\title{
FEMTOSECOND LIGHT WAVE PACKETS
}

\section{RADZEWICZ}

Institute of Experimental Physics, Warsaw University, Hoża 69, 00-681 Warsaw, Poland

\author{
M. Trippenbach, Y.B. Band
}

Department of Chemistry and Physics, Ben-Gurion University, Beer Sheva, Israel

\author{
AND J.S. KRASINSKI
}

Center for Laser and Photonics Research, Oklahoma State University

Stillwater, OK, 74078, USA

We analyze propagation of ultra short light pulses in a transparent, dispersive, nonlinear medium. A general formula for femtosecond wave packet evolution is developed and applied to specific problems. Theoretical and experimental results for wave packet distortion by lenses, wave packet rotation in birefringent media and group velocity matching in sum frequency generation are presented. Numerical results for splitting of femtosecond wave packets in dispersive Kerr media are also presented.

PACS numbers: 42.65.Jx, 42.65.Re

\section{Introduction}

Developments in laser mode-locking techniques during the last decade led to generation of pulses as short as $7.5 \mathrm{fs}\left(1 \mathrm{fs}=10^{-15} \mathrm{~s}\right)$ directly from Ti:sapphire laser [1]. By employing pulse compression techniques one can achieve even shorter pulses with the current record at less than 5 fs level $[2,3]$. Progress in this field has been very fast, especially in the 70-ties and 80-ties, as illustrated in Fig. 1 which presents the history of ultra short pulse generation in the last 25 years. Originally, the most common femtosecond systems were based on organic dyes as laser materials, however with an advent of fs Ti:sapphire lasers [4] in the early 90-ties a solid state technology is available. Currently, systems operating at $20-30$ fs range are routine and commercial systems with sub-20 fs capabilities are on the market.

A femtosecond light pulse propagating in a given medium can be visualized as a wave packet of electro-magnetic radiation that moves with its group velocity and at the same time changes its shape due to the interaction with the medium. We find the wave packet picture appealing and we will use the wave packet language throughout this paper. One of the simplest experiments with femtosecond light pulses involves propagation of such wave packets through a transparent medium, 


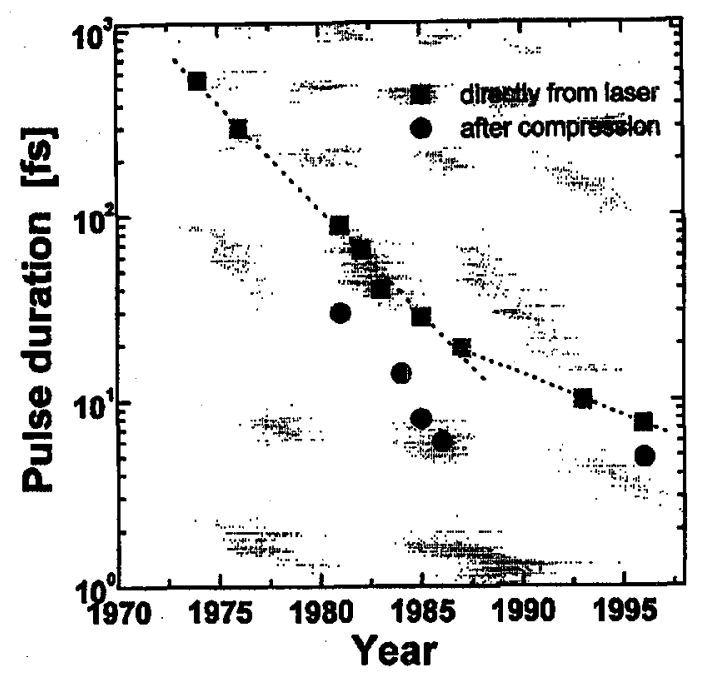

Fig. 1. A history of ultra short light pulse generation. Pulse duration available directly from lasers and after compression are shown.

for example an optical window made of glass. Such experiments are omnipresent. They are performed every day in ultra fast optics laboratories, often without a conscious thought on the experimentalist part, whenever an ultrashort light pulse has to be delivered from the laser system to the sample and, on its way, passes through a lens or a glass cell window. Since the result of many experiments with ultra short light pulses, especially in a nonlinear regime, strongly depends on the details of the pulse such as its time and/or spatial properties it is quite important to understand what happens to such a pulse on its way from the laser system to the sample. When considering propagation of a low intensity monochromatic light beam in a dielectric one only has to account for its index of refraction which modifies the diffraction of the beam by changing the propagation constant with respect to that for vacuum. What makes the propagation of femtosecond pulses different? First, by the virtue of their brevity, the femtosecond pulses have broad spectrum. Because of that, a significant pulse shaping is observed whenever they propagate in a dispersive medium. Second, the peak intensity can be quite high even with a moderate pulse energy and average power. Therefore 3-rd order processes (allowed in a medium of any symmetry) cannot be neglected. For instance, intensity dependent refraction index gives rise to self-phase modulation and self-focusing which influence the spectrum of the pulse and its spatio-temporal characteristics, respectively. It is a well-known phenomenon leading, among other things, to formation of optical solitons in fibers [5, 6] and white light continuum generation [7]. As is often the case in nonlinear problems, three phenomena: diffraction, dispersion and self-focusing are entangled; one can study their effects separately only in a limited number of cases when one of them dominates. However, in most cases numerical methods have to be employed as a method of solution. This paper is organized as follows. In Sec. 2 we present a general form of the propagation equation for a fem- 
tosecond wave packet in a transparent, dispersive and nonlinear medium. Section 3 provides examples of the wave packet shaping effects in a linear regime - both theoretical and experimental data are provided. In Sec. 4 we analyze nonlinear propagation regime and present the results of numerical calculations illustrating a complex wave packet evolution.

\section{Propagation equation}

We start by representing the scalar electric field $E(r, t)$ of the light wave packet as an integral of its Fourier components

$$
E(r, t)=\frac{1}{(2 \pi)^{3}} \int \mathrm{d}^{3} k \tilde{E}(k) \exp \{[k \cdot r-\omega(k) t]\},
$$

each characterized by its amplitude $\widetilde{E}(k)$, wave vector $k$ and frequency $\omega(k)$. Out of four variables, i.e. frequency and three components of the wave vector that describe each Fourier component, only three are independent because of the dispersion relation $\omega=\omega(k)$. Next we represent the field amplitude as a product of a slowly varying envelope (SVE) and a phase factor

$$
E(r, t)=A(r, t) \exp \left\{\mathrm{i}\left[k_{0} \cdot r-\omega\left(k_{0}\right) t\right]\right\},
$$

where we assumed that the wave vectors are grouped around a central value $k_{0}$ and correspondingly the frequencies form a band around the central frequency $\omega_{0}=\omega\left(k_{0}\right)$. This leads to the propagation equation for slowly varying envelope $[8-11]$

$$
\begin{aligned}
& \frac{\partial A(r, t)}{\partial z}=\frac{1}{(2 \pi)^{3}} \int_{-\infty}^{+\infty} \mathrm{d}^{3} k A(k, \omega)\left(\mathrm{i}\left[k-k_{0}\right] \cdot s_{0}\right) \\
& \quad \times \exp \left\{\mathrm{i}\left[\left(k-k_{0}\right) \cdot r-\left(\omega-\omega_{0}\right) t\right]\right\}+\mathrm{i} \gamma_{n l}|A|^{2} A .
\end{aligned}
$$

In the last equation a unit length vector $s_{0}=k_{0} /\left|k_{0}\right|$ assumed to be in the direction of $z$ coordinate has been introduced. An additional term $\mathrm{i} \gamma_{n l}|A|^{2} A$ has also been added. It describes the effect of the 3-rd order nonlinearity (Kerr type nonlinearity) of the medium. The integral over $k_{z}$ can be replaced by an integral over $\omega$ when the dispersion relation is taken into account:

$$
k_{z}=\sqrt{\omega^{2} n^{2}(\omega, s) / c^{2}-k_{x}^{2}-k_{y}^{2}},
$$

where $n$ is the index of refraction and $s=k /|k|$. This particular form of expression for $n$ allows application of this approach to both isotropic and anisotropic media. Since no assumptions about the form of $A(r, t)$ have been made Eq. (3) is exact. A partial differential equation for $A(r, t)$ can also be obtained by using Eq. (3). This is done by expanding $k_{z}$ in powers of $k_{x}, k_{y}$ and $\left(\omega-\omega_{0}\right)$ and replacing these variables with $\mathrm{i} \partial / \partial x, \mathrm{i} \partial / \partial y$ and $-\mathrm{i} \partial / \partial t$, respectively. Keeping terms up to the second order gives for a uniaxial birefringent medium [11]

$$
\begin{aligned}
\frac{\partial A}{\partial z} & =-\beta_{1} \frac{\partial A}{\partial t}+\frac{\mathrm{i}}{2} \beta_{2} \frac{\partial^{2} A}{\partial t^{2}}+\gamma_{x} \frac{\partial A}{\partial x}+\mathrm{i} \gamma_{t x} \frac{\partial^{2} A}{\partial t \partial x} \\
& +\frac{\mathrm{i}}{2} \gamma_{x x} \frac{\partial^{2} A}{\partial x^{2}}+\frac{\mathrm{i}}{2} \gamma_{y y} \frac{\partial^{2} A}{\partial y^{2}}+\mathrm{i} \gamma_{n l}|A|^{2} A .
\end{aligned}
$$


The coefficients $\beta_{1}=1 / \nu_{\mathrm{g}}=\partial k / \partial \omega$ and $\beta_{2}=\partial \beta_{1} / \partial \omega$ describe the effects of group velocity and group velocity dispersion, respectively, $\gamma_{x x}$ and $\gamma_{y y}$ are responsible for the diffraction of the wave packet, $\gamma_{x}$ reflects beam walk-off in a birefringent medium while $\gamma_{t x}$ was found to be responsible for the rotation of the wave packet [9]. It is worth mentioning that the coefficients $\gamma_{x}$ and $\gamma_{t x}$ vanish for isotropic medium. One can easily generalize Eq. (4) by adding higher order terms. For the sake of clarity we will not do it here but rather refer an interested reader to the original papers [8-11]. Either of the two equations (Eq. (3) or the expanded version of Eq. (4)) can be numerically integrated. It should be pointed out that Eq. (4) is an approximation of Eq. (3) and in order to make them totally equivalent one would have to include terms of all orders into Eq. (4). This clearly is not a practical approach. We found however that in all cases studied, the results of either approach are almost identical if the terms up to 3-rd order are included into Eq. (4). Before we present the results of such integration let us consider some simpler cases.

\section{Linear propagation: 1-D and 3-D effects}

First, let us analyze Eq. (4) in a 1-dimensional case. Assume that $A(r, t)=$ $A(z, t)$. This is the case whenever light propagates in a single mode optical fiber or the laser beam properties are such that it can be modeled as a one-dimensional plane wave. In this case all derivatives with respect to $x$ and $y$ vanish and we are left with a well-known nonlinear Schrödinger equation of the following form:

$$
\frac{\partial A}{\partial z}=-\beta_{1} \frac{\partial A}{\partial t}+\frac{\mathrm{i}}{2} \beta_{2} \frac{\partial^{2} A}{\partial t^{2}}+\mathrm{i} \gamma_{\mathrm{nl}}|A|^{2} A .
$$

This equation is easy to solve if we keep only the first term on the right side. One can verify that any function $A(z, t)$ that has a form $A\left(z, z / \nu_{\mathrm{g}}\right)$ constitutes a proper solution. This means that in the lowest order approximation a 1-dimensional wave packet can be of any shape and it propagates without distortions with the speed equal to the group velocity in a given medium. It may seem to be a trivial statement, which it is, but a one with significant consequences in many experiments. We will illustrate this with two examples.

The first example of the havoc that the group velocity can play with an experiment is a wave packet distortion by lenses. It has been recognized quite a long time ago that the difference between phase and group velocities can lead to significant effects upon propagation of femtosecond pulses through lenses [12-13]. If a collimated beam, i.e. a flat wave packet, is focused with a chromatic lens then the phase surfaces behind the lens are spherical but the shape of the wave packet is not. In the UV-visible range the group velocity is smaller than the phase velocity for optical glasses. Because the wave packet propagates through more glass in the center of the beam where the lens is the thickest it accumulates more group delay than phase delay there while at the edges of the lens the two delays are almost equal. As a result the wave packet behind the lens is distorted; its central part lags behind the edges. This in turn causes that the intensity of light in the focus no longer follows the intensity time profile of the input pulse because different radial zones of the wave packet contribute to it at different times. It is important to be able to measure this distortion, especially for complex lenses such 


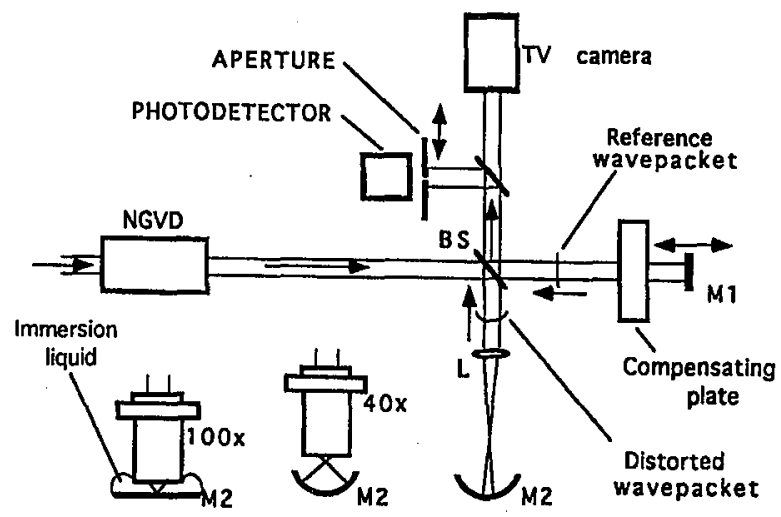

Fig. 2. An experimental set-up used to measure wave packet distortion by lenses. NGVD is a negative group velocity dispersion prismatic line. $L$ is the tested lens. The TV camera has been used to align the interferometer.

as microscope objectives for which direct calculations may be quite difficult and which are commonly used in applications such as two-photon microscopy [14]. We have designed an interferometric method to measure the wave packet distortion in lenses [15]. The experimental set-up is shown in Fig. 2. The main part of the system is a modified Michelson interferometer with one arm serving as a reference arm and the other arm including the tested lens $L$. A collimated beam from a femtosecond Ti:sapphire laser was used as an input beam. Its diameter was big enough to fill the aperture of the lens. As indicated in the figure, at the output of the interferometer a flat reference wave packet interferes with a wave packet distorted by a double passage through the lens. The interference fringes are visible only in the regions where the two wave packets overlap for a given delay defined by the position of the mirror $M 1$. As this mirror is scanned the fringes appear and disappear in different radial zones of the output beam. A detector placed behind a small pinhole was used to measure the fringe visibility at different distances from the beam center. For any given position of the pinhole we measured the delay corresponding to maximum fringe visibility and thus found the shape (we assume cylindrical symmetry) of the distorted wave packet. An example of the results is presented in Fig. 3. It shows the relative group delay as a function of the radial position on a $40 \times$ microscope objective. As one can see the parts of the wave packet propagating close to the edges of the lens are advanced by more than $30 \mathrm{fs}$ with respect to the center of the wave packet. We found it quite amusing that an addition of a cover glass ( $170 \mu \mathrm{m}$ thick) between the lens and the curved mirror $M 2$ significantly improved the performance of the objective. Apparently the objective has been designed to work with the cover glass!

As a second example, consider a frequency mixing experiment with fermtosecond laser pulses. A crystal with $\chi^{(2)}$ nonlinearity is illuminated by two collinear ultra short laser pulses with frequencies $\omega_{1}$ and $\omega_{2}$ and a sum frequency at $\omega_{3}=\omega_{1}+\omega_{2}$ is generated. Since the three frequencies involved in the process are quite different, the group velocities of the corresponding pulses are differ- 


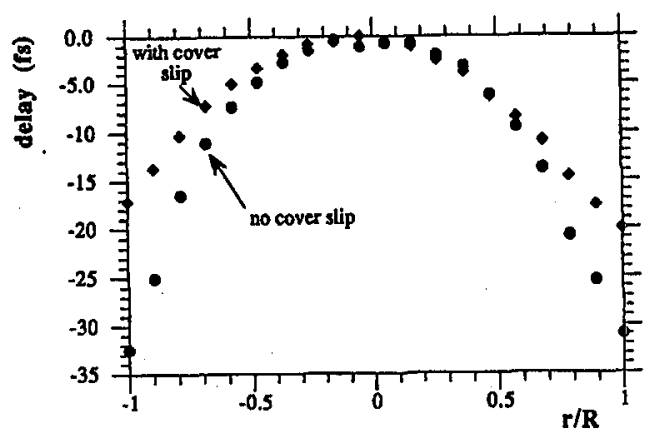

Fig. 3. Wave packet distortion by $40 \times, 0.65$ NA Olympus microscope objective. Relative delays for different radial zones of the wave packet are shown.

ent, too. This has a detrimental effect on the sum frequency generation process. If $\omega_{1} \neq \omega_{2}$ then $\nu_{\mathrm{g}}\left(\omega_{1}\right) \neq \nu_{\mathrm{g}}\left(\omega_{2}\right)$ in a dispersive medium. As a result the two input pulses propagate with different speeds. This limits the range over which the input pulses overlap which in turn limits the efficiency of the process. In addition, the output pulse at $\omega_{3}$ propagates at yet another (and usually very different) speed. As a result, the output pulse is longer than the input pulses because contributions from different slices of the crystal arrive at the output face at different times. The latter effect remains even in the case of second harmonic generation (SHG) when a single femtosecond pulse is used to produce another pulse at twice the input frequency. The problem of group velocity mismatch could be significantly alleviated if a frequency mixing scheme which ensures both phase matching (PM) and group velocity matching (GVM) could be found. We found that this can be actually achieved in some cases when type I non-collinear sum frequency generation scheme is applied $[16,17]$.

Figure 4 illustrates the basic idea. In the UV-visible range the group velocity in nonlinear crystals decreases with increasing frequency and thus the sum frequency pulse at $\omega_{3}$ lags behind the driving pulses at $\omega_{1}$ and $\omega_{2}$. Therefore with a suitable choice of the angles $\psi_{1}$ and $\psi_{2}$ the projections of $\nu_{\mathrm{g} 1}, \boldsymbol{\nu}_{\mathrm{g} 2}$ and $\nu_{\mathrm{g} 3}$ on $k_{3}$ can be made equal. This means that the three pulses have the same components of the group velocity along the propagation direction and they do not separate as they propagate. It is not obvious that group velocity matching and phase matching can be achieved in a given crystal for given wavelengths of the input pulses. Whether this is possible or not depends on dispersion properties of the particular nonlinear birefringent crystal selected. Figure 5 shows the results of numerical calculations for $\beta$-barium borate (BBO) crystal in the range of fundamental wavelengths corresponding to the tunability range of Ti:sapphire femtosecond oscillator. Two processes have been considered: non-collinear type I SHG $(\omega+\omega \rightarrow 2 \omega)$ and non-collinear type I third harmonic generation (THG) $(\omega+2 \omega \rightarrow 3 \omega)$. The two cases are quite different. For SHG the problem involves solving two equations (phase matching condition and group velocity matching condition) for two variables $\psi=\psi_{1}=\psi_{2}$ and $\Theta\left(\Theta\right.$ is an angle between $k_{3}$ and the optic axis of the crystal) and the solutions are exact if they exist. In the case of THG there are 


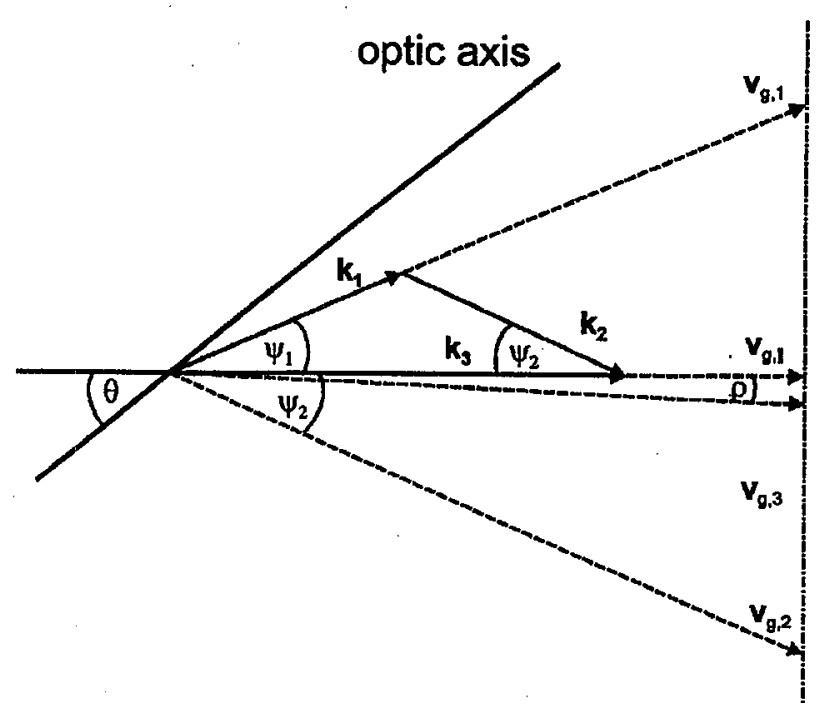

Fig. 4. A schematic diagram of non-collinear phase matching and group velocity matching scheme for sum frequency generation. The angles $\psi_{1}$ and $\psi_{2}$ are chosen so that the components of the group velocity of all three waves along the $k$ vector of the output signal are equal. $\Theta$ is the phase matching angle.
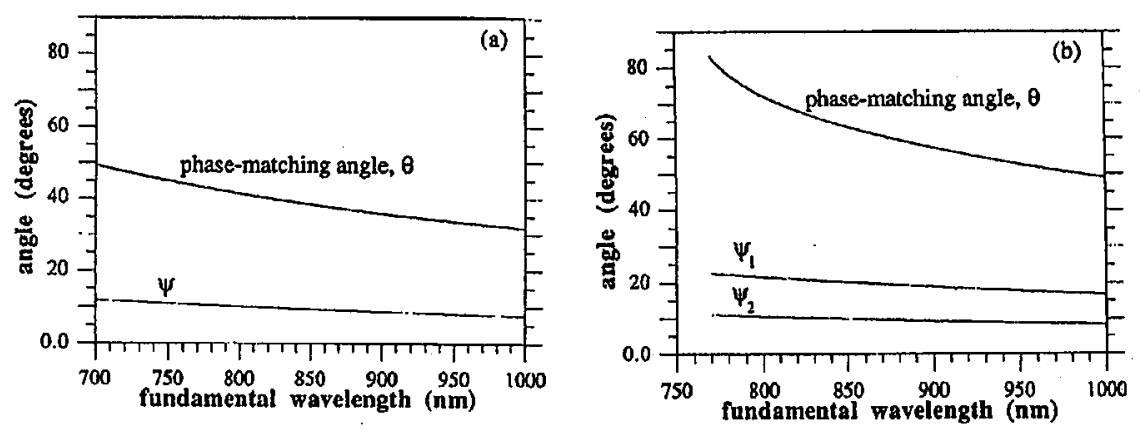

Fig. 5. (a) Phase-matching angle $\Theta$ and angle $\psi$ for SHG in BBO versus wavelength. (b) Phase-matching angle $\Theta$ and angles $\psi_{1}$ and $\psi_{2}$ for THG in BBO versus wavelength. Note that for THG group velocity matching is possible only for wavelengths longer than $740 \mathrm{~nm}$.

3 variables $\psi_{1}, \psi_{2}$, and $\Theta$ and only two equations. Phase matching condition has to be fulfilled but then group velocity matching is not exact. However, the results of numerical calculations indicate that the residual group velocity mismatch in our scheme can be as small as one percent of that for a standard collinear THG case. As one can see in Fig. 5 PM and GVM conditions can be achieved for SHG in BBO over an entire tuning range of Ti:sapphire laser. Similarly exact PM and approximate GVM are possible for THG process in a somewhat smaller range of wavelengths. 
In order to see the effect of GVM on frequency mixing with femtosecond pulses we have numerically integrated the nonlinear equations for the amplitudes of the three fields

$$
\begin{aligned}
& \frac{\partial A_{1}}{\partial z}+\frac{\beta_{21}}{2} \frac{\partial^{2} A_{1}}{\partial t^{2}}=-\frac{\mathrm{i} \omega_{1}}{n_{1}} \chi A_{3} A_{2}^{*}, \\
& \frac{\partial A_{2}}{\partial z}+\Delta \beta_{12} \frac{\partial A_{2}}{\partial t}+\frac{\beta_{22}}{2} \frac{\partial^{2} A_{2}}{\partial t^{2}}=-\frac{\mathrm{i} \omega_{2}}{n_{2}} \chi A_{3} A_{1}^{*}, \\
& \frac{\partial A_{3}}{\partial z}+\Delta \beta_{13} \frac{\partial A_{3}}{\partial t}+\frac{\beta_{23}}{2} \frac{\partial^{2} A_{3}}{\partial t^{2}}=-\frac{\mathrm{i} \omega_{3}}{n_{3}} \chi A_{1} A_{2},
\end{aligned}
$$

where $A_{i}$ is the amplitude, $\omega_{i}$ - the frequency, $n_{i}$ - the refraction index of the $i$-th field and $\beta_{2 i}=\partial^{2} k_{i} / \partial \omega^{2}$. A reference frame moving with the group velocity of the $\omega_{1}$ pulse is used and $\Delta \beta_{1 i}=1 / \nu_{\mathrm{g} i}-1 / \nu_{\mathrm{g} 1}$ for $i=2,3$.

The results of the integration are shown in Fig. 6 . One can clearly see that for reasonable experimental conditions the effect of group velocity mismatch is not negligible. Second harmonic pulses in a GVM non-collinear scheme can be significantly shorter than those achievable in a standard collinear scheme.

This has been verified in an experiment performed with approximately $30 \mathrm{fs}$ long pulses from a Ti:sapphire laser and a $0.5 \mathrm{~mm}$ long BBO crystal. The second harmonic has been generated using two different approaches: a standard collinear
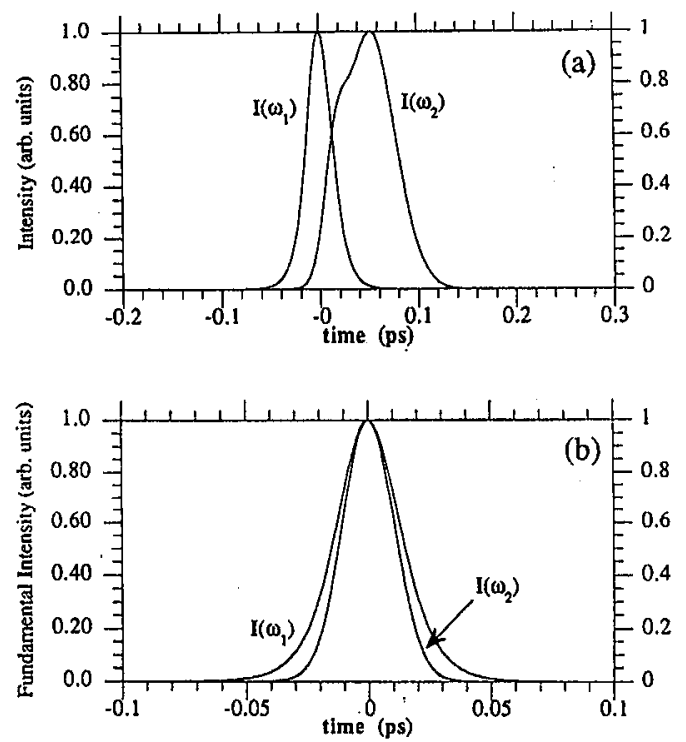

Fig. 6. Numerical results for SHG in a $0.5 \mathrm{~mm}$ thick BBO crystal. Input pulse duration is $30 \mathrm{fs}$ and input wavelength $800 \mathrm{~nm}$, (a) shows fundamental and second harmonic pulses for collinear geometry while (b) shows the same data for non-collinear (GVM) scheme. Note that in (b) the second harmonic pulse is shorter than the laser pulse. 
SHG and a non-collinear GVM SHG. In each case the duration of the second harmonic pulse has been measured by intensity cross-correlation with the input laser pulse. Results of the cross-correlation measurements are shown in Fig. 7. For the standard collinear scheme the output pulse duration is almost twice of that for the laser pulse. At the same time the results show that for the GVM scheme second harmonic pulses that are shorter than the input laser pulses, can be achieved. This is not surprising since in an ideal case of SHG without saturation one should expect the second harmonic pulse to be shorter than the input pulse by a factor of $\sqrt{2}$.

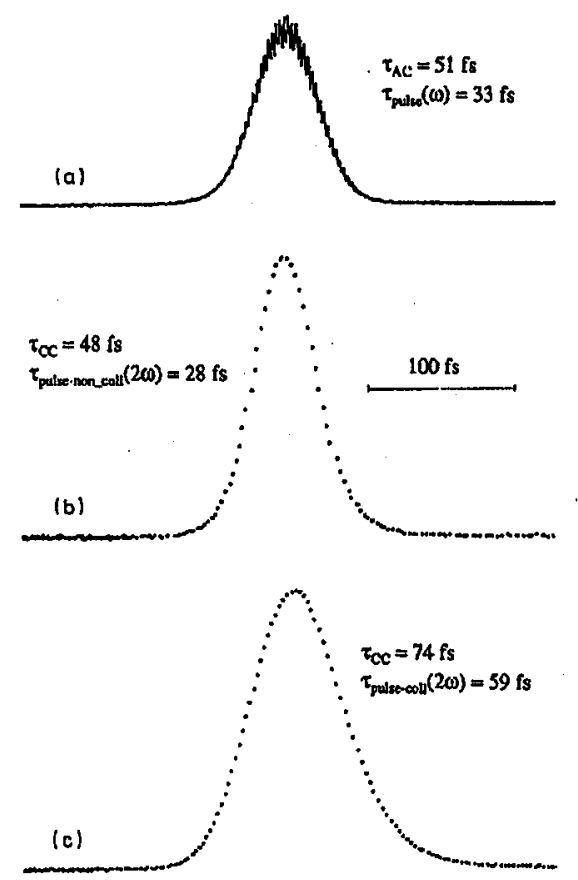

Fig. 7. Experimental results for (a) the auto-correlation of the fundamental pulse, (b) the cross-correlation of the fundamental with the $\mathrm{SH}$ pulse in the non-collinear experiment, and (c) the cross-correlation of the fundamental pulse with the SH pulse in the collinear experiment.

As a final example of linear propagation effects let us consider a femtosecond wave packet propagating in a birefringent dispersive medium. If the light propagates as an extraordinary wave then the terms $\gamma_{x}$ and $\gamma_{t x}$ in Eq. (4) are non-zero. While the first one means that the Poynting vector in such a medium is not parallel to the wave vector (a fact known for at least a century) the existence and meaning of the second one have been found and explained only recently $[8,9]$. Numerical integration of Eq. (4) shows that because of this term the wave packet, which is tightly focused when it enters an uniaxial birefringent crystal, rotates around the axis that is perpendicular to the plane defined by optic axis of the crystal and the $k$ vector. The effect can be explained in a way very similar to that employed to explain the wave packet distortion by lenses. Because of the tight focusing the ex- 
panding beam contains wave vectors that form different angles with the optic axis of the crystal. Since, for an extraordinary wave, the index of refraction depends on this angle, both phase and group velocities are also functions of the same angle. What is even more important, the difference between phase velocity and group velocity varies as a function of this angle. As a result, one side of the wave packet lags more behind the phase fronts than the other side which leads to the wave packet rotation. If such a pulse is recollimated after it exits the crystal, it will look skewed - while the phase fronts will be perpendicular to the direction of propagation, the wave packet itself will be not, simply because the difference between phase and group delays will be different at its opposite sides. The theoretical predictions for the wave packet rotation have been verified experimentally in a set-up [18] similar to the one shown in Fig. 2. The set-up has been modified to include two identical 1:1 telescopes, one in each arm. Two $1 \mathrm{~mm}$ thick rutile crystals have been placed, one at the focus of each telescope. The wave packets propagating in the crystals experience rotation as described above. We have placed the crystals in both arms to cancel all the distortions of the wave packets that are not due to the rotation in the crystal. However the crystals were set in such a way that the two wave packets experienced rotation in opposite directions and thus did not overlap perfectly in space. By recording the fringe visibility at different positions in the output beam versus the delay between two arms of the interferometer we were able to measure the wave packet rotation.

The results of the experiment (points) are compared to the theoretically calculated wave packet rotation (line) in Fig. 8. It is clear that our model for wave packet rotation is at least adequate as indicated by the agreement of its predictions with the experimental results.

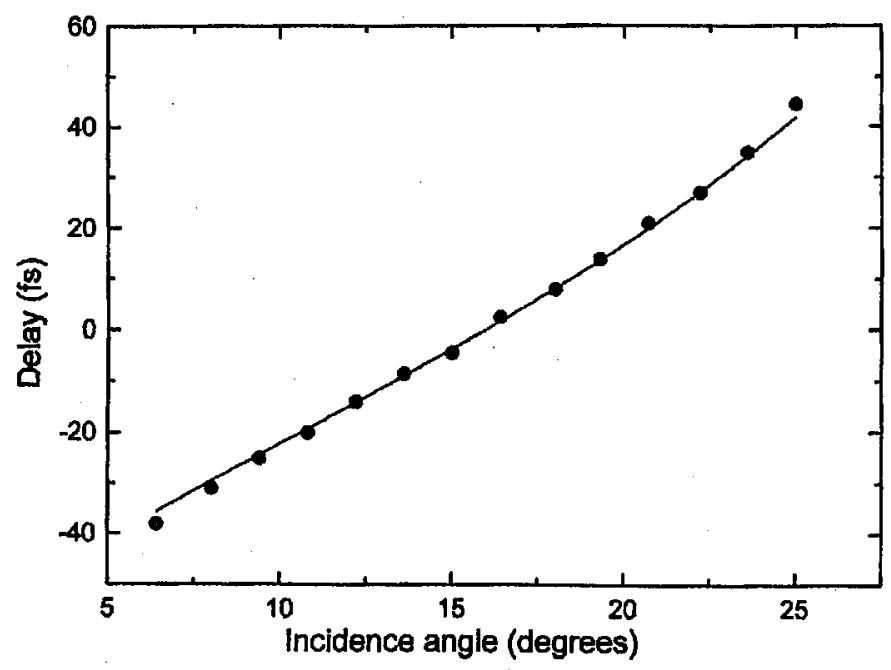

Fig. 8. Measured (dots) and calculated (line) wave packet rotation in rutile crystal. 
To complete our analysis of the linear propagation we should briefly mention the effects of the group velocity dispersion. When the group velocity dispersion term - (i/2) $\beta_{2} \partial^{2} A / \partial t^{2}$ is also included into the analysis of Eq. (5) the pulse shape does not remain constant during propagation. Instead, because different Fourier components of the pulse experience different group delays, the result is a pulse with varying shape and time dependent frequency. In this approximation the light frequency varies linearly with time and thus the pulse is said to have a linear chirp. The effect can be quite severe. For instance, a 25 fs long Fourier limited pulse at $630 \mathrm{~nm}$ doubles its duration upon propagation through $10 \mathrm{~mm}$ of fused silica. The reshaping of femtosecond pulses due to propagation in dispersive media means that in any given experiment we cannot take the pulse duration for granted. Even if we verify that the pulse is short when it exits the laser system, it can be quite longer when it interacts with our sample simply because there was some glass on the way from the laser to the interaction region! An addition of higher order dispersion terms leads to even more significant pulse reshaping and a nonlinear chirp.

\section{Nonlinear propagation}

Let us start again with 1-dimensional case. The nonlinear term i $\gamma_{\mathrm{n} 1}|A|^{2} A$ in Eq. (5) describes the effect of intensity dependent index of refraction $n(A)=$ $n_{0}+\gamma_{n 1}|A|^{2}$. This introduces a time dependent phase and nonlinear chirp without affecting the pulse shape. As a result, new frequencies are generated and the pulse spectrum broadened. Spectrum broadening in Kerr media can be combined with a group velocity dispersion to produce pulses that are significantly shorter than the original laser pulses in a technique called pulse compression [19]. When both group velocity dispersion and Kerr nonlinearity terms are included the net result depends on the signs of $\beta_{2}$ and $\gamma_{\mathrm{nl}}$ coefficients. If the two terms have the same sign the chirp due to dispersion and the one due to Kerr nonlinearity have the same sign, too. As a result the pulse experiences reshaping and a combined chirp. On the other hand, if $\operatorname{sgn}\left(\beta_{2}\right)=-\operatorname{sgn}\left(\gamma_{\mathrm{nl}}\right)$ then there exist stable solutions of the propagation equation [5]. Such solutions are called optical solitons. Because they can propagate over long distances in optical fibers without changes in shape they are of particular interest to engineers designing optical communication systems. In one of the experiments in this field picosecond pulses have been propagated in a fiber of length $1.8 \times 10^{11} \mathrm{~m}$, i.e. further than from the Earth to the Sun [20].

In a 3-D case the interplay between dispersion and nonlinearity is augmented by an additional effect, namely a competition between diffraction and self-focusing. While the first tends to increase the transverse dimensions of the wave packet, the latter does exactly the opposite. It has been known for at least 30 years [21] that for long pulses the ultimate fate of a beam propagating in a Kerr medium is determined by its power $P$. For $P<P_{c}\left(P_{\mathrm{c}}\right.$ is called critical power and depends on the medium properties only) diffraction wins and the beam defocuses. If, however, $P>P_{\mathrm{c}}$ self-focusing prevails and catastrophic beam collapse is observed. This is not true for femtosecond pulses. As has been pointed out by several groups [22-25] in this case one has to take into account dispersion effects as well. Because of that the wave packet with a power higher than the critical power does not collapse; 
instead it undergoes a complex evolution both in time and space. In order to illustrate this evolution let us turn back to Eq. (4).

For a wave packet that is Gaussian both in time and transverse spatial distribution propagating in an isotropic medium, it is convenient to rewrite Eq. (4) in a slightly different form [11]

$$
\frac{\partial A}{\partial z}=-\beta_{1} \frac{\partial A}{\partial t}-\mathrm{i} \frac{\tau_{0}^{2}}{L_{\mathrm{ds}}} \frac{\partial^{2} A}{\partial t^{2}}+\mathrm{i} \frac{w_{0}^{2}}{L_{\mathrm{df}}}\left(\frac{\partial^{2} A}{\partial x^{2}}+\frac{\partial^{2} A}{\partial y^{2}}\right)+\mathrm{i} \frac{1}{\left|A_{0}\right|^{2} L_{\mathrm{nl}}}|A|^{2} A
$$

where three new parameters all of length dimension have been introduced: dispersion length $L_{\mathrm{ds}}=\tau_{0}^{2} / \beta_{2}$, diffraction length $L_{\mathrm{df}}=\gamma_{x x} w_{0}^{2} / 2=\pi w_{0}^{2} / \lambda_{0}$, and nonlinear length $L_{\mathrm{nl}}=\left(\gamma_{\mathrm{nl}}\left|A_{0}^{2}\right|\right)^{-1}$ with $\tau_{0}, w_{0}$, and $A_{0}$ being the duration, transverse size, and amplitude of the wave packet, respectively. The advantage of such scaling is that it provides three parameters that can be easily compared to each other which in turn enables one to evaluate the strength of the three phenomena they represent (the smaller the coefficient the more important the corresponding term in Eq. (7). The meaning of these parameters is as follows: $L_{\mathrm{ds}}$ is a distance
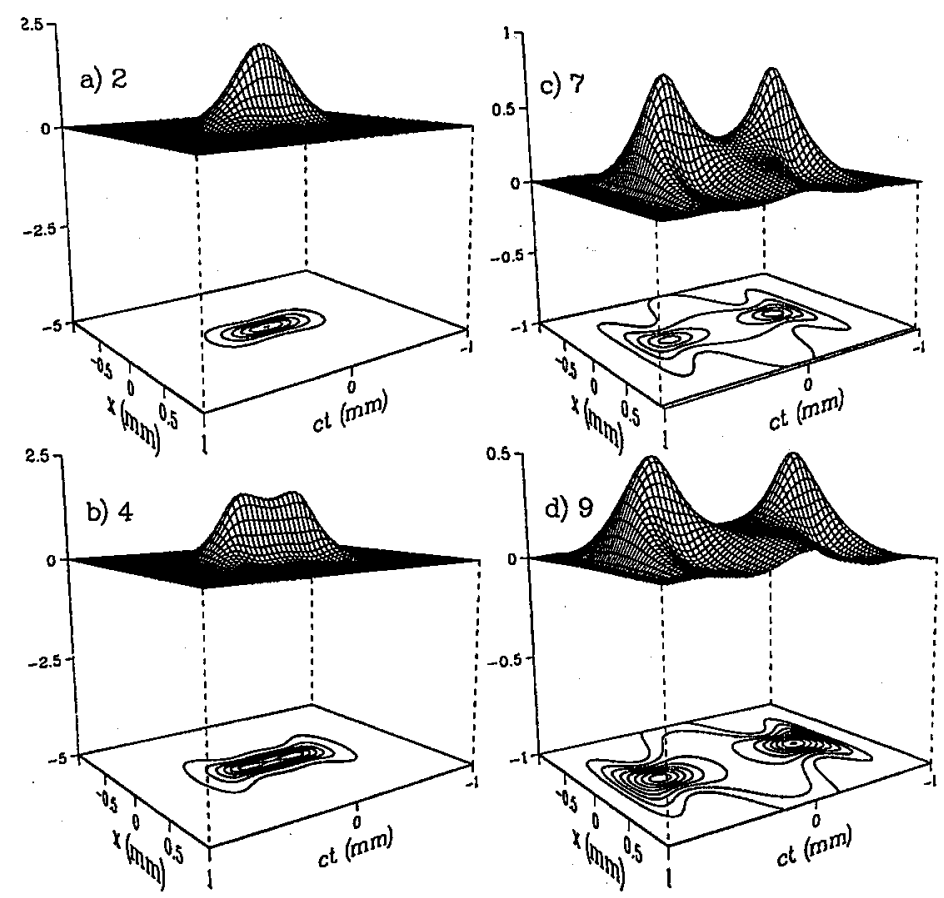

Fig. 9. Numerical results for nonlinear propagation of a wave packet in fused silica. Numbers in each figure indicate the distance that the wave packet has propagated inside the medium. 

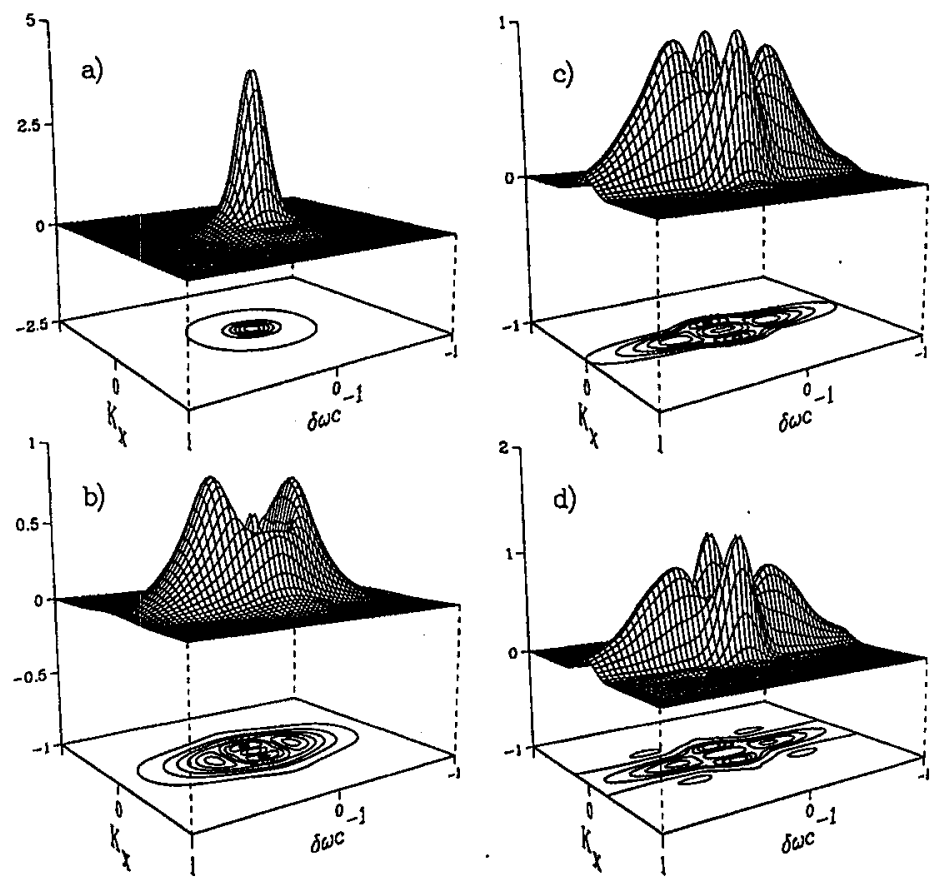

Fig. 10. Spatio-temporal spectrum of the wave packets shown in Fig. 9. $K_{x}$ is the spatial frequency in the $x$ direction.

which takes the pulse to double its duration because of the medium dispersion, $L_{\mathrm{df}}$ is the distance required for the pulse to double its area as a result of diffraction, and $L_{\mathrm{nl}}$ is the self-focusing distance. For transparent media the inequality $L_{\mathrm{ds}} \gg L_{\mathrm{nl}}, L_{\mathrm{df}}$ typically holds. It means that the dispersion effects constitute only a small correction to the basic wave packet evolution defined by diffraction and self-focusing. However, the results of integration of Eq. (7) show that, small as it is, the dispersion plays a crucial role in propagation of a femtosecond wave packet. An example of the wave packet evolution obtained by direct integration of Eq. (3) is shown in Fig. 9 (the results were very similar when Eq. (7) with 3-rd order terms included was integrated). In this particular case it was assumed that the wave packet size in the $y$ direction is much larger than its size in the $x$ direction. This not only made the computation simpler but also enabled us to present the results as a 3-D graphs. The following parameters have been assumed: material fused silica $\left(L_{\mathrm{ds}}=240 \mathrm{~mm}\right)$, pulse duration $-66 \mathrm{fs}$, pulse wavelength $-800 \mathrm{~nm}$, beam size $-32 \mu \mathrm{m}\left(L_{\mathrm{df}}=4 \mathrm{~mm}\right)$, pulse intensity $-70 \mathrm{GW} / \mathrm{cm}$ ? $\left(L_{\mathrm{nl}}=2 \mathrm{~mm}\right)$. The power of the beam is about $2.2 P_{c}$. A long pulse of this power would self-focus and collapse. However the femtosecond wave packet considered here displays a quite different behavior. It starts to self-focus as indicated by the elongated shape in Fig. 9a but then the process is arrested and the pulse splits into two pulses as shown in Figs. 9c and d. It is instructive to look at the spatio-temporal spectrum of the wave packet shown in Fig. 10. Starting from a smooth Guassian shape the 
spectrum evolves through a "Mexican hat" structure (Fig. 10c) and then develops three spatial lobes. The central lobe (around $k_{x}=0$ ) contains two peaks in the frequency distribution, one at the frequencies lower than the input pulse frequency and another which is shifted towards higher frequencies. The two side lobes have broad spectra centered at the input pulse frequency. The results depend, as one might expect for a nonlinear problem, on a particular choice of parameters, for example the sign and magnitude of the $\beta_{2}$ coefficient, but from the numerical integration results some general conclusions about the role of the dispersion can be drawn. For the case illustrated in Figs. 9 and 10 the process starts with the self-focusing. The self-focusing is the strongest at $c t=0$ (Fig. 9a) simply because the intensity is the highest there. Thus the intensity at the center of the wave packet increases rapidly and so does the width of spectrum due to self-phase modulation. Once the spectrum is broad enough the dispersion shifts lower frequencies towards the head of the pulse and higher frequencies towards its tail. This lowers the intensity at the center of the wave packet and prevents it from a collapse. At the same time the part of the wave packet that has been focused strongly diffracts to form the side lobes in the spectrum as discussed above. The pulse splitting shown in Fig. 9 has been recently observed in an experiment [26].

In conclusion, we have analyzed some aspects of linear and nonlinear propagation of femtosecond light wave packets in transparent, dispersive nonlinear media. In particular, we have shown the effects of group velocity on propagation of such wave packets through lenses, wave packet rotation in birefringent media and sum frequency generation without group velocity mismatch. Numerical results showing wave packet splitting were also presented.

\section{References}

[1] L. Xu, Ch. Spielmann, F. Krausz, R. Szipocs, Opt. Lett. 21, 1259 (1996).

[2] M. Nisoli, S. De Silvestri, O. Svelto, R. Szipocs, F. Ferencz, Ch. Spielmann, S. Sartania, F. Krausz, Opt. Lett. 22, 522 (1997).

[3] A. Baltuska, Z. Wei, M.S. Pshenichnikov, D.A. Wiersma, Opt. Lett. 22, 102 (1997).

[4] D.E. Spence, P.N. Kean, W. Sibbet, Opt. Lett. 16, 42 (1991).

[5] V.E. Zakharov, A.B. Shabat, Sov. Phys. JETP 34, 62 (1970).

[6] L.F. Mollenauer, R.H. Stolrn, J.P. Gordon, Phys. Rev. Lett. 45, 1095 (1980).

[7] For example: The Supercontinuum Laser Source, Ed. R.R. Alfano, Springer-Verlag, New York 1989.

[8] Y.B. Band, M. Trippenbach, Phys. Rev. Lett. 76, 1457 (1996).

[9] M. Trippenbach, Y.B. Band, J. Opt. Soc. Am. B 13, 1403 (1996).

[10] M. Trippenbach, T.C. Scott, Y.B. Band, Opt. Lett. 22, 579 (1997).

[11] M. Trippenbach, Y.B. Band, Phys. Rev. A 56, 1 (1997).

[12] H. Staerk, J. Ihlemann, A. Hembold, Laser Optoelectronic 20, 6 (1988).

[13] Z. Bor, Opt. Lett. 14, 119 (1989).

[14] P.F. Curley, A.I. Ferguson, J.G. White, W.B. Amos, Optical Quantum Electron. 24, 851 (1992). 
[15] C. Radzewicz, M.J. la Grone, J.S. Krasinski, Opt. Commun. 126, 185 (1996).

[16] C. Radzewicz, Y.B. Band, G.W. Pearson, J.S. Krasinski, Opt. Commun. 117, 295 (1995).

[17] Y.B. Band, M. Trippenbach, C. Radzewicz, J.S. Krasinski, J. Nonlinear Opt. Phys. Mater. 5, 477 (1996).

[18] C. Radzewicz, J.S. Krasinski, M.J. la Grone, M. Trippenbach, Y.B. Band, J. Opt. Soc. $A m . B$ 14, 420 (1997).

[19] C.V. Shank, R.L. Fork, R. Yen, R.H. Stolen, W.J. Tomlinson, Appl. Phys. Lett. 40, 761 (1982).

[20] M. Nakazawa, K. Suzuki, E. Yamada, H. Kubota, Y. Kimura, in: Proc. Conf. on Fiber Communications (OFC'93), Opt. Soc. Am., Washigton (D.C.) 1993, WC1.

[21] Y.R. Shen, The Principles of Nonlinear Optics, John Wiley \& Sons, New York 1984.

[22] D. Strickland, P.B. Corkum, Proc. Soc. Photo-Opt. Instrum. Engl. 1413, 54 (1991).

[23] P. Chernev, V. Petrov, Opt. Lett. 17, 172 (1992).

[24] J.E. Rothenberg, Opt. Lett. 17, 172 (1992).

[25] G.G. Luther, A.C. Newell, J.V. Moloney, E.M. Wright, Opt. Lett. 19, 789 (1994).

[26] J.K. Ranka, R.W. Schirmer, A.L. Gaeta. Phys. Rev. Lett. 77, 3783 (1996). 